\title{
Molecular evolution of two consecutive carotenoid cleavage dioxygenase genes in strigolactone biosynthesis in plants
}

\author{
R.K. Wang, J.J. Lu, G.N. Xing, J.Y. Gai and T.J. Zhao \\ National Key Laboratory for Crop Genetics and Germplasm Enhancement, \\ National Center for Soybean Improvement, Soybean Research Institute, \\ Nanjing Agricultural University, Nanjing, P.R. China \\ Corresponding author: T.J. Zhao \\ E-mail: tjzhao@njau.edu.cn
}

Genet. Mol. Res. 10 (4): 3664-3673 (2011)

Received May 1, 2011

Accepted October 24, 2011

Published December 2, 2011

DOI http://dx.doi.org/10.4238/2011.December.2.2

\begin{abstract}
Strigolactones are newly discovered plant hormones that perform various functions, from signaling in symbiotic interactions with arbuscular mycorrhizal fungi to controlling outgrowth of axillary buds. We examined the phylogenetic relationships of two carotenoid cleavage dioxygenase genes $(C C D 7$ and $C C D 8)$ that are involved in consecutive upstream steps of the proposed strigolactone biosynthesis pathway. The CCD7 and CCD 8 sequences from 11 model species, divided into two clades, correspond to sequences from monocotyledons and dicotyledons. However, the sequences from the primitive moss, Physcomitrella patens, appeared to be evolutionarily distinct from those of the angiosperms. $C C D 7$ and $C C D 8$ are much conserved, since no significant positive selection was detected among these plants. $K s$ values indicated that $C C D 7$ and $C C D 8$ diverged about 290 to 430 million years ago. As essential genes in the strigolactone pathway, the divergence timing of the conserved $C C D 7$ and $C C D 8$ genes reflects the approximate time of generation of strigolactone as a regulatory substance. This timing calculation also coincides with initiation of symbiosis between plants and microorganisms, inferred from
\end{abstract}


the fossil record. Molecular evolution analyses of genes in metabolic pathways can provide insight concerning gene evolution.

Key words: Strigolactones; Carotenoid cleavage dioxygenase; Phylogenetic comparison; Gene divergence; Symbiosis

\section{INTRODUCTION}

Strigolactones are a group of terpenoid lactones found in root exudates of diverse plant species. They are characterized as seed germination stimulants of root parasitic plants, such as Striga sp and Orobanche sp (Umehara et al., 2008), and they act as root-derived signals for symbiotic interactions with arbuscular mycorrhizal fungi (AM), which promote the absorption of nutrients from the soil by plants (Yokota et al., 1998; Besserer et al., 2006). Such symbioses are observed in more than $80 \%$ of terrestrial plants, which coincides with the wide distribution of this class of terpene compounds (Keeling et al., 2010). AM are incapable of completing their life cycle in the absence of a host root. While, AM spores can germinate and grow in the absence of a host, actual hyphal growth is very limited. The first fossils containing land plants and fungi together are from 460 to 480 million years ago (Mya) (Redecker et al., 2000; Heckman et al, 2001).

The full characterization of the strigolactone biosynthetic pathway is underway. Recent studies indicate that strigolactones are derived from carotenoids, and the pathway of strigolactone biosynthesis contains two conserved carotenoid cleavage enzymes (CCDs), which are encoded by the carotenoid cleavage dioxygenase genes $C C D 7$ and $C C D 8$. Some target genes have been cloned and studied in Arabidopsis, pea and other plants (Drummond et al., 2009; Vogel et al., 2010). Among these genes MAX3, RMS5 and HTD1/D17 are CCD7s, while MAX4, RMS1, D10 and DAD1 are CCD8s (Matusova et al., 2005; Foo et al., 2007). $C C D 7$ and $C C D 8$ are consecutive genes in the strigolactone pathway, and they have been shown to be evolutionarily closely related (Drummond et al., 2009).

Based on nucleotide conservation, the rate of DNA sequence substitution can serve as a molecular clock to infer the divergence times of extant organisms (Ayala, 1977). In plant evolution, there are non-synonymous and synonymous nucleotide substitutions. Synonymous substitutions are not influenced by Darwinian selection, and they are always used for molecular clock measurements (Tamura et al., 2007). On the other hand, positive selection can be detected by non-synonymous substitutions and the ratio of non-synonymous substitutions to synonymous substitutions (dN/dS) (Yang, 2007). CCD7 and CCD8 play key roles in the strigolactone biosynthesis pathway. In the present study, phylogenetic relationships of $C C D 7$ and $C C D 8$ in various plants were analyzed to infer evolutionary trends and divergence timing.

\section{MATERIAL AND METHODS}

\section{Identification and structure prediction of the $C C D$ sequences in plants}

The amino acid sequences of CCD7 and CCD8 for Arabidopsis thaliana (AtCCD7 and AtCCD8; NCBI numbers 130064.4 and 119434.3, respectively) were used as the reference sequences, and BLASTP was performed using the Phytozome website (http://www. phytozome.net). A total of 11 model species were selected for sequence analyses of their $C C D 7$ 
and $C C D 8$ genes, including five monocotyledons, five dicotyledons and a moss (Table 1). The $C C D 7$ and $C C D 8$ genes were identified according to their E-values in the BLASTP program and synteny to other $C C D$ s. This syntenic information was collected from Phytozome. The longest sequence was selected in gene clusters or multiple transcriptions.

To illustrate exon/intron structure of the $C C D$ genes, the Gene Structure Display Server program (http://gsds.cbi.pku.edu.cn/index.php) was used to compare the genomic DNA to their corresponding cDNA sequences on the Phytozome website.

\section{Phylogenetic analyses}

Amino acid sequence alignments were performed using the ClustalW module in the MEGA (Molecular Evolutionary Genetics Analysis) 4.0 program (Tamura et al., 2007; Morrison, 2007). Neighbor-joining trees and bootstrap analyses were also conducted using MEGA 4.0, and the following parameters were selected: model, p-distance; bootstrap, 1000 replicates; and gap/missing data, pairwise deletion.

\section{Positive selection analyses}

Protein sequences of the $C C D$ gene pairs were aligned in MEGA 4.0 (Tamura et al., 2007), and the results were used to guide the coding sequence (CDS) alignments by Pal2Nal (http://www. bork.embl.de/pal2nal/). Positive selection can be detected using a maximum likelihood approach with the Codeml procedure in the PAML 4.0 software (Yang, 2007), under the situations of site model, branch model (Yang, 1998) and branch-site model (Yang and Nielsen, 2002). The likelihood ratio test can detect whether the model is significantly better than the null one.

\section{Calculating number of synonymous substitutions per site $(K s)$ and divergence time of $C C D 7$ and $C C D 8$}

The number of synonymous substitutions per site $(K s)$ was determined using the CDS aligned by the neighbor-joining method. This was implemented in the phylogenetic analysis by PAML 4.0 after all alignment gaps had been eliminated (Yang, 2007). The Ks values of CCD7 and $C C D 8$ between monocotyledon and dicotyledon sequences were calculated separately.

To estimate the divergence timing of $C C D 7$ and $C C D 8$, the date of the monocotyledondicotyledon divergence was used as a reference. This date was selected for a number of reasons. First, the divergence timing of the two $C C D$ s is within one order of magnitude, which can reduce the system error. Second, the divergence of monocotyledons and dicotyledons is widespread in all the angiosperms. Finally, much research on fossils and molecular evolution has focused on this time (Chaw et al., 2004). The synonymous substitution rate $(\lambda)$ was calculated according to the formula $T=K s / 2 \lambda$ (Rensing et al., 2007), where $T$ is the estimated divergence time of $C C D 7-C C D 8$.

\section{RESULTS}

\section{Identification and structure prediction of the $C C D$ sequences in plants}

BLASTP was performed to identify homology and syntenic information for the plant 
$C C D$ sequences. The sequences with lowest E-values were identified (Table 1). The amino acid length of the sequences ranged from 823 to 2,002 residues. The exon/intron organization was conserved in all these $C C D$ s. The number of exons in $C C D 7$ ranged from 6 to 9, while in $C C D 8$ there were 5 or 6 exons in dicotyledon species and 3 to 5 exons in monocotyledons (Figure 1).

\begin{tabular}{|c|c|c|c|c|c|c|c|}
\hline Gene name & Gene code & Species name & Class & $\begin{array}{l}\text { Gene start } \\
\text { position (bp) }\end{array}$ & $\begin{array}{l}\text { Gene end } \\
\text { position (bp) }\end{array}$ & $\begin{array}{c}\text { Gene } \\
\text { length (bp) }\end{array}$ & $\begin{array}{c}\text { Protein } \\
\text { length (aa) }\end{array}$ \\
\hline PtrCCD7 & POPTR_0014s05590 & Populus trichocarpa & Dicot. & 4172126 & 4176634 & 4508 & 602 \\
\hline GmaCCD7 & Glyma1̄ing16370 & Glycine $\max$ & Dicot. & 12178329 & 12184335 & 6006 & 620 \\
\hline PpeCCD7 & ppa017865m.g & Prunus persica & Dicot. & 15623254 & 15626943 & 3689 & 617 \\
\hline AthCCD7 & AT2G44990 & Arabidopsis lyrata & Dicot. & 18558938 & 18561621 & 2683 & 631 \\
\hline VviCCD7 & GSVIVG01018217001 & Vitis vinifera & Dicot. & 13131131 & 13135439 & 4308 & 612 \\
\hline SbiCCD7 & Sb06g024560 & Sorghum bicolor & Monocot. & 53677260 & 53679729 & 2469 & 623 \\
\hline $\mathrm{ZmaCCD7}$ & GRMZM2G158657 & Zea mays & Monocot. & 19458968 & 19461625 & 2657 & 626 \\
\hline SitCCD7 & Si009605m.g & Setaria italica & Monocot. & 26555134 & 26558032 & 2898 & 633 \\
\hline OsaCCD7 & LOC_Os04g46470 & Oryza sativa & Monocot. & 27382685 & 27385787 & 3102 & 611 \\
\hline BdiCCD7 & Bradi5g 17660 & Brachypodium distachyon & Monocot. & 20891073 & 20893960 & 2887 & 639 \\
\hline PpaCCD7* & Pp1s14_421V6 & Phycomitrella patens & Bryophyta & 2624330 & 2627670 & 3340 & 695 \\
\hline PtrCCD8 & POPTR_0006s25490 & Populus trichocarpa & Dicot. & 23860613 & 23864595 & 3982 & 559 \\
\hline GmaCCD8 & Glyma04 $\mathrm{g} 08910$ & Glycine $\max$ & Dicot. & 7056680 & 7060578 & 3898 & 551 \\
\hline PpeCCD8 & ppa006042m.g & Prunus persica & Dicot. & 36886170 & 36889062 & 2892 & 433 \\
\hline AthCCD8 & AT4G32810 & Arabidopsis lyrata & Dicot. & 15828228 & 15831485 & 3257 & 572 \\
\hline VviCCD8 & GSVIVG01035626001 & Vitis vinifera & Dicot. & 2785955 & 2788687 & 2732 & 565 \\
\hline SbiCCD 8 & Sb03g034400 & Sorghum bicolor & Monocot. & 62608453 & 62611997 & 3544 & 581 \\
\hline ZmaCCD8 & GRMZM2G446858 & Zea mays & Monocot. & 197015856 & 197019350 & 3494 & 598 \\
\hline SitCCD8 & $\mathrm{Si} 000867 \mathrm{~m} . \mathrm{g}$ & Setaria italica & Monocot. & 36666879 & 36671129 & 4250 & 562 \\
\hline OsaCCD 8 & LOC_Os01g38580 & Oryza sativa & Monocot. & 21664573 & 21668264 & 3691 & 554 \\
\hline BdiCCD8 & Bradi2g49670 & Brachypodium distachyon & Monocot. & 49700664 & 49704240 & 3576 & 572 \\
\hline PpaCCD8* & Pp1s14_419V6 & Phycomitrella patens & Bryophyta & 2616379 & 2619648 & 3269 & 584 \\
\hline
\end{tabular}

The genes for $C C D 7$ and $C C D 8$ are the typical names in Phytozome database. *Sequences that are absent in syntenic regions.

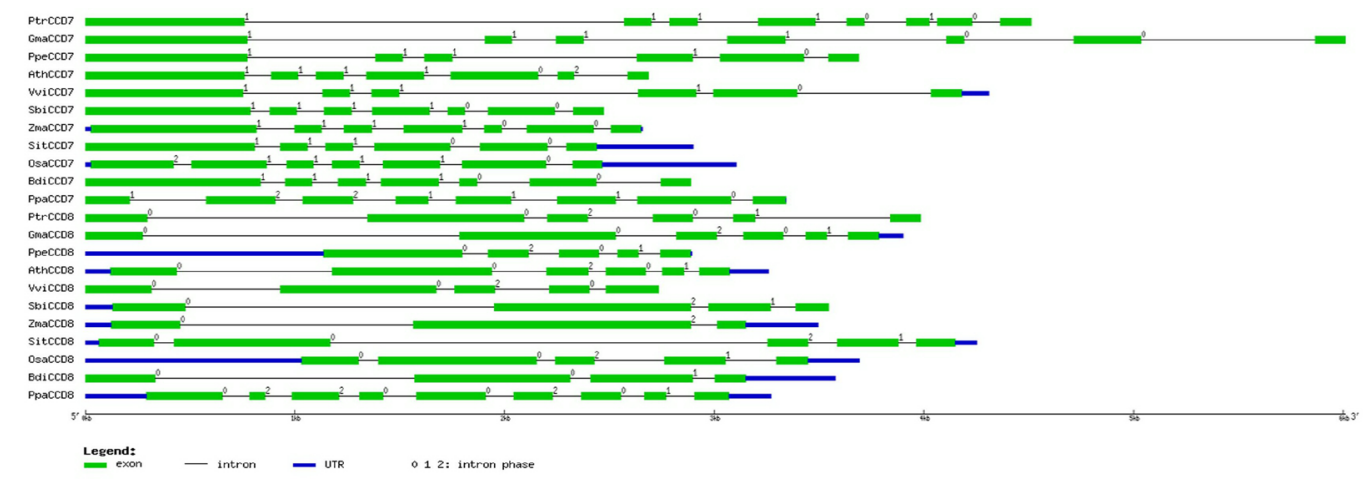

Figure 1. The exon/intron structures of the collective $C C D$ genes. Boxes represent the exons and lines represent the introns. The sizes of exons and introns can be estimated using the scale at bottom of the figure. The numbers above the boxes and lines indicate the splicing phases of the $C C D$ sequences: 0 , phase $0 ; 1$, phase $1 ; 2$, phase 2 .

\section{Phylogenetic analyses of $C C D 7$ and $C C D 8$ genes}

BLASTP was performed with the amino acid sequences and neighbor-joining trees prepared using corresponding nucleotide sequences. Since amino acid sequences are more 
conserved than the nucleotide sequences, a phylogenetic tree based on amino acid sequences divided the $C C D 7$ and $C C D 8$ genes into distinct groups (Figure 2). Each of these groups contained two clusters corresponding to monocotyledon and dicotyledon species. $C C D 7$ and $C C D 8$ had similar evolutionary trends, and the greatest distance appeared to be between the moss (P. patens) and all the angiosperm species (Figure 2). This finding indicated that $C C D 7$ and $C C D 8$ had already diverged in this primitive moss.

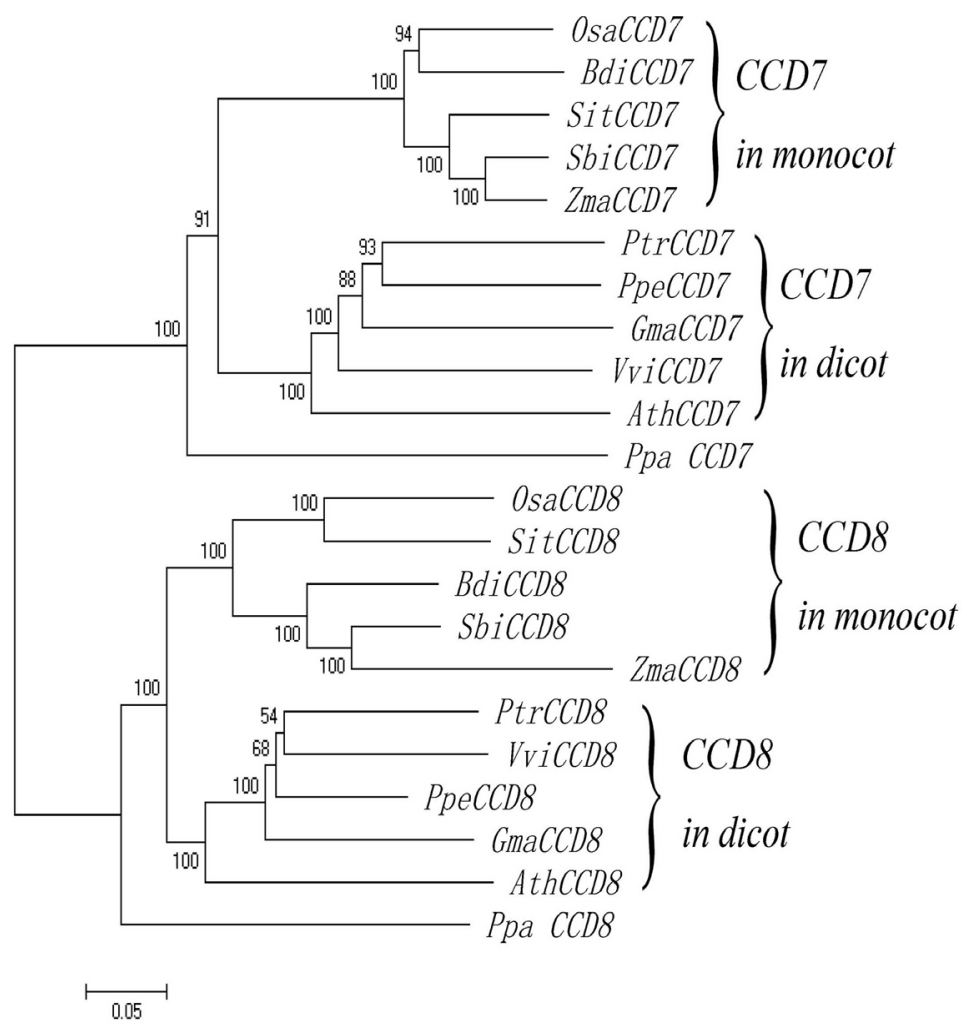

Figure 2. Phylogenetic analyses of $C C D 7$ and $C C D 8$. The trees were constructed using the neighbor-joining method in MEGA 4.0. The numbers beside the branches represent bootstrap values ( $>50 \%)$ based on 1,000 replicates.

\section{Positive selection analyses of $C C D 7$ and $C C D 8$}

In the site model, all the branches are considered to have the same non-synonymous/ synonymous rate ratio (dN/dS, denoted $\omega)$, and two pairs of models, i.e., M1a (neutral) versus M2a (selection) and M7 (beta) versus M8 (beta and $\omega$ ), are used to detect positive sites (Zhang et al., 2005). The M2a and M8 models did not differ significantly from the null model (Table 2), indicating that the CCDs are rather conserved. In the branch model of two ratios, there were differences in the $\mathrm{dN} / \mathrm{dS}$ ratios of foreground compared to background branches. Branches of dicotyledon $C C D 7$ and monocotyledon $C C D 8$ had greater $\omega_{1}$ in their foreground branches, indicating possible selection pressure on these genes. The branch of dicotyledon $C C D 8$ was the most conserved $\left(\omega_{0}=0.13, \omega_{1}=0.07\right)$. 
Table 2. Summary statistics for detecting selection.

\begin{tabular}{|c|c|c|}
\hline Model & $\ln L$ & Parameter estimates \\
\hline M0 & -15846.83 & $\omega_{0}=0.11$ \\
\hline M1a & -15783.45 & $\begin{aligned} p_{0} & =0.94, p_{1}=0.06 \\
\omega_{0} & =0.11, \omega_{1}=1.00\end{aligned}$ \\
\hline M1b & -15783.45 & $\begin{array}{l}p_{0}=0.94, p_{1}=0.02, p_{2}=0.04 \\
\omega_{0}=0.11, \omega_{1}=1.00, \omega_{2}=1.00\end{array}$ \\
\hline M7 & -15672.07 & $p=1.33, q=8.89$ \\
\hline M8 & -15671.04 & $\begin{array}{l}p_{0}=0.99, p=1.40, q=9.70 \\
p_{1}=0.01, \omega_{1}=1.00\end{array}$ \\
\hline \multicolumn{3}{|l|}{ Branch model } \\
\hline Monocot CCD7 & -15846.79 & $\omega_{0}=0.11, \omega_{1}=0.12$ \\
\hline Dicot CCD7 & $-15838.09^{* *}$ & $\omega_{0}=0.10, \omega_{1}=0.15$ \\
\hline Monocot CCD8 & $-15823.02 * *$ & $\omega_{0}=0.09, \omega_{1}=0.22$ \\
\hline Dicot CCD8 & $-15831.13^{* *}$ & $\omega_{0}=0.13, \omega_{1}=0.07$ \\
\hline \multicolumn{3}{|l|}{ Branch-site } \\
\hline Monocot CCD7-Null & -15747.09 & $\begin{array}{l}p_{0}=0.83, p_{1}=0.04, p_{2}+p_{3}=0.13 \\
\omega_{0}=0.10, \omega_{1}=\omega_{2}=1.00\end{array}$ \\
\hline Monocot CCD7 & -15747.09 & $\begin{array}{l}p_{0}=0.83, p_{1}=0.04, p_{2}+p_{3}=0.13 \\
\omega_{0}=0.10, \omega_{2}=1.00\end{array}$ \\
\hline Monocot CCD8-Null & -15778.51 & $\begin{array}{l}p_{0}=0.84, p_{1}=0.05, p_{2}+p_{3}=0.11 \\
\omega_{0}=0.10, \omega_{1}=\omega_{2}=1.00\end{array}$ \\
\hline Monocot CCD8 & -15778.51 & $\begin{array}{l}p_{0}=0.84, p_{1}=0.05, p_{2}+p_{3}=0.11 \\
\omega_{0}=0.10, \omega_{2}=1.00\end{array}$ \\
\hline Dicot CCD7-Null & -15711.89 & $\begin{array}{l}p_{0}=0.82, p_{t}^{2}=0.03, p_{2}+p_{3}=0.16 \\
\omega_{0}=0.09, \omega 1=\omega_{2}=1.00\end{array}$ \\
\hline Dicot CCD7 & -15711.89 & $\begin{array}{l}p_{0}=0.82, p_{1}=0.03, p_{2}+p_{3}=0.16 \\
\omega_{0}=0.09, \omega_{2}=1.00\end{array}$ \\
\hline Dicot CCD8-Null & -15781.95 & $\begin{array}{l}p_{0}=0.93, p_{1}^{2}=0.06, p_{2}+p_{3}=0.02 \\
\omega_{0}=0.11, \omega_{1}=\omega_{1}=1.00\end{array}$ \\
\hline Dicot CCD8 & -15783.45 & $\begin{array}{l}p_{0}=0.94, p_{1}=0.06, p_{2}+p_{3}=0 \\
\omega_{0}=0.11, \omega_{2}=1.00\end{array}$ \\
\hline
\end{tabular}

395 amino acids were used in PAML 4.0 after all gaps were deleted. PpaCCD7 and PpaCCD 8 were not used in this step. **extremely significant in likelihood ratio test (P-value less than 0.01$)$.

The branch-site model assumes that the $\omega$ ratio varies between codon sites and that there are four site classes in the sequence according to $\omega$ values (Wolfe et al., 1989b; Zhang et al., 2005). The two subfamilies of $C C D s$ perform different biochemical functions. However, in the branch-site model, different clades had no selection sites or branches, so the two $C C D$ subfamilies appear to have similar evolutionary character. Therefore, no positive selection was detected and the two $C C D s$ appeared to be rather well conserved.

\section{$K s$ values of $C C D 7$ and $C C D 8$}

$K s$ values for the 20 sequences (P. patens was not included) were obtained using the Codeml program (Table 3). In the phylogenetic tree of $C C D$ evolution (Figure 3), two divergences appear to have occurred. The first divergence was between $C C D 7$ and $C C D 8$, while the second was between the monocotyledons and dicotyledons. The $K s$ value between monocotyledon $C C D 7$ and dicotyledon $C C D 8$ was $4.89 \pm 0.30$, and between dicotyledon $C C D 7$ and monocotyledon $C C D 8$ this was $5.25 \pm 0.41$. The two values were calculated using pairs of sequences that had sustained both divergence events. These data mark the divergence time of $C C D 7-C C D 8$. In the $C C D 7$ or $C C D 8$ clades, the average $K s$ values between monocotyledons and dicotyledons were $2.89 \pm 0.06$ and $2.74 \pm 0.26$, respectively. These data mark the monocotyledon-dicotyledon divergence time. 
R.K. Wang et al.

3670

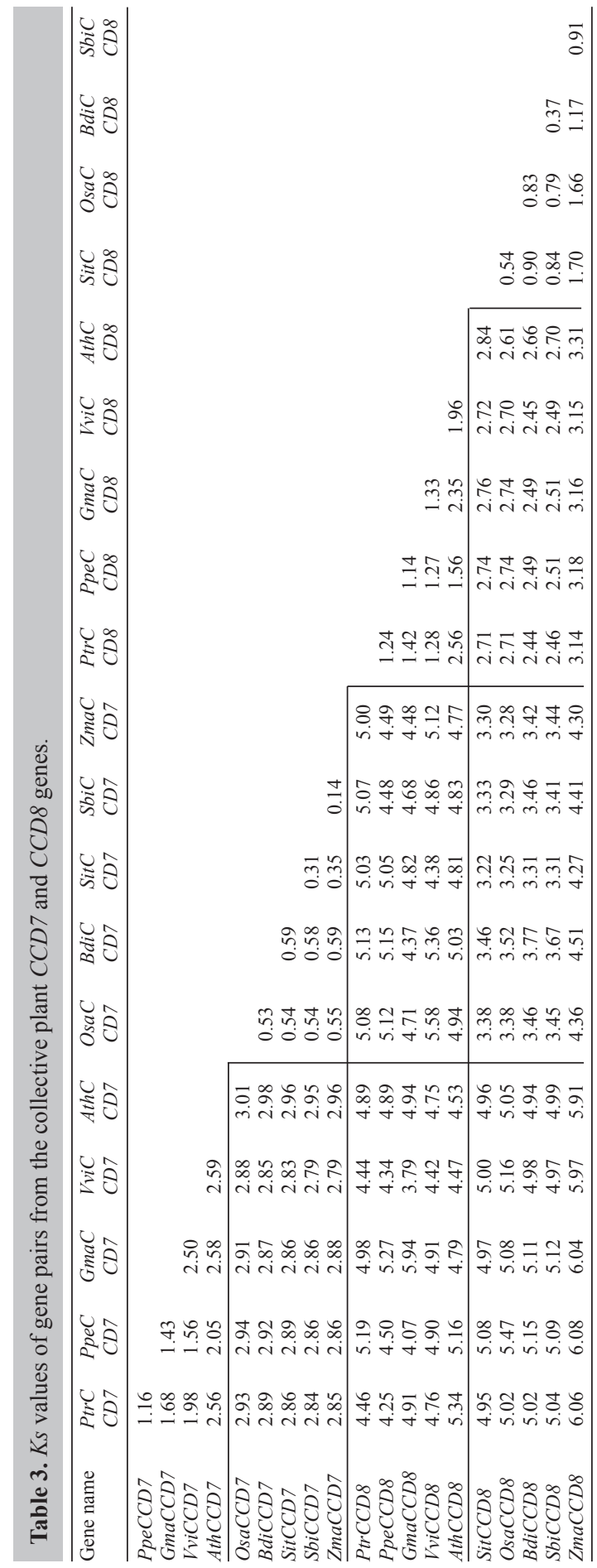




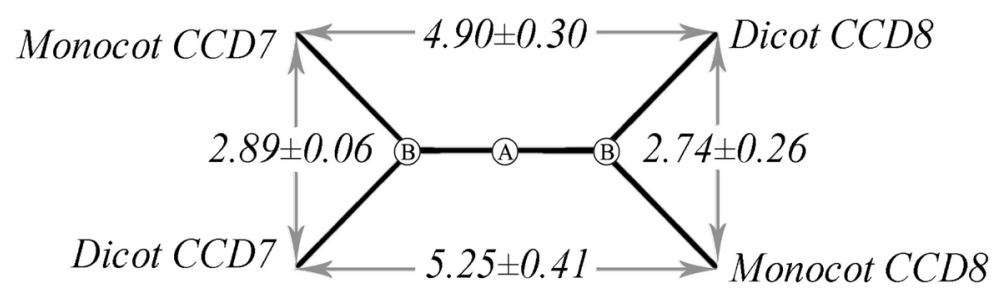

Figure 3. Simple phylogenetic tree of $C C D 7$ and $C C D 8$ in angiosperms. The numbers are the $K s$ values, which indicate the evolutionary difference. A stands for the divergence event of CCD7 and CCD8, and B stands for the divergence event of monocotyledons and dicotyledons.

Molecular dating often assumes substitution rate constancy, but this is frequently violated (Wolfe et al., 1989b; Chaw et al., 2004). Sanderson and Doyle (2001) considered that much of the conflict in estimating divergence times was due to rate variation across lineages. In the present study, the similar Ks-value pairs between $C C D 7$ and $C C D 8(4.89 \pm 0.30$ to 5.25 $\pm 0.41)$ and within monocotyledons and dicotyledons $(2.89 \pm 0.06$ to $2.74 \pm 0.26)$ were similar, indicating constant substitution rate of the branches.

\section{Estimation of divergence time of $C C D 7$ and $C C D 8$}

Differences existed in the nuclear synonymous substitution values of $C C D s$ between monocotyledons and dicotyledons. To reduce variation, average $K s$ values from each monocotyledon-dicotyledon comparison were used (Figure 3). The divergence time of CCD7-CCD8 was 1.59 to 2.04 times the monocotyledon-dicotyledon divergence time.

Many studies have focused on the time of divergence of monocotyledons and dicotyledons and results vary between 140 to 350 Mya (Chaw et al., 2004). In the present study, the date of about 200 Mya with an uncertainty of about 40 Mya presumed by Wolfe et al. (1989a) was used, and the divergence time of $C C D 7-C C D 8$ was estimated to be 1.8 times the monocotyledon-dicotyledon divergence time, giving a date of the $C C D 7-C C D 8$ divergence between 290 to 430 Mya. The synonymous substitution rate $(\lambda)$ was estimated to be $7.04 \times 10^{-9}$ site/year.

\section{DISCUSSION}

The dN/dS ratios of the $C C D$ genes were small (Table 2), and positive selection was not obvious, meaning that most sequence substitutions were neutral. Moreover, sequence homology was detected between a bryophyte and angiosperm plants (E-value to A. thaliana below $1 \times 10^{-100}$ ). Therefore, $C C D 7 s$ and $C C D 8 s$ could be similar in structure to their ancestors. Two CCDs and strigolactone have been found in the moss Physcomitrella patens (Keeling et al., 2010; Proust et al., 2011), so the divergence time of the two CCDs was before the divergence time of mosses and angiosperms, which occurred 370 Mya (Wolfe et al., 1989a).

Hitherto, some CCD proteins for apocarotenoid synthesis have been identified, and these proteins exhibit specificity for the double bond they cleave. $C C D 7$ and $C C D 8$ catalyze similar biosynthetic reactions to other $C C D s$ in that they perform carotenoid cleavage steps in the strigolactone pathway. No other functions of $C C D 7$ and $C C D 8$ have been identified in plants (Auldridge et al., 2006). Thus, ancestral CCD7 and CCD8 proteins may have per- 
formed the same functions as the contemporary proteins, and the divergence times of $C C D 7$ and $C C D 8$ may have occurred just after the strigolactone generation time. Strigolactones are signaling compounds in symbiotic interactions between AM and plants. These symbioses play significant roles in aquatic and terrestrial plants (Redecker et al., 2000; Heckman et al, 2001; Umehara et al., 2008). Fossil data show that fungi and plants may have started such symbioses about 460 to 480 Mya. The data in the present study is in accordance with this date.

\section{ACKNOWLEDGMENTS}

Research supported by the National Key Basic Research Program (\#2010CB1259 and \#2009CB1184), the Natural Science Foundation of China (\#31071442), the MOA Special Project for Crop Breeding (\#2011ZX08004-001) and the MOE 111 Project (\#B08025).

\section{REFERENCES}

Auldridge ME, McCarty DR and Klee HJ (2006). Plant carotenoid cleavage oxygenases and their apocarotenoid products. Curr. Opin. Plant Biol. 9: 315-321.

Ayala FJ (1977). Nothing in biology makes sense except in the light of evolution. J. Hered. 68: 3-10.

Besserer A, Puech-Pages V, Kiefer P, Gomez-Roldan V, et al. (2006). Strigolactones stimulate arbuscular mycorrhizal fungi by activating mitochondria. PLoS Biol. 4: e226.

Chaw SM, Chang CC, Chen HL and Li WH (2004). Dating the monocot-dicot divergence and the origin of core eudicots using whole chloroplast genomes. J. Mol. Evol. 58: 424-441.

Drummond RS, Martinez-Sanchez NM, Janssen BJ, Templeton KR, et al. (2009). Petunia hybrida CAROTENOID CLEAVAGE DIOXYGENASE7 is involved in the production of negative and positive branching signals in petunia. Plant Physiol. 151: 1867-1877.

Foo E, Morris SE, Parmenter K, Young N, et al. (2007). Feedback regulation of xylem cytokinin content is conserved in pea and Arabidopsis. Plant Physiol. 143: 1418-1428.

Heckman DS, Geiser DM, Eidell BR, Stauffer RL, et al. (2001). Molecular evidence for the early colonization of land by fungi and plants. Science 293: 1129-1133.

Keeling CI, Dullat HK, Yuen M, Ralph SG, et al. (2010). Identification and functional characterization of monofunctional ent-copalyl diphosphate and ent-kaurene synthases in white spruce reveal different patterns for diterpene synthase evolution for primary and secondary metabolism in gymnosperms. Plant Physiol. 152: 1197-1208.

Matusova R, Rani K, Verstappen FWA, Franssen MCR, et al. (2005). The strigolactone germination stimulants of the plant-parasitic Striga and Orobanche spp. are derived from the carotenoid pathway. Plant Physiol. 139: 920-934.

Morrison DA (2007). Increasing the efficiency of searches for the maximum likelihood tree in a phylogenetic analysis of up to 150 nucleotide sequences. Syst. Biol. 56: 988-1010.

Proust H, Hoffmann B, Xie X, Yoneyama K, et al. (2011). Strigolactones regulate protonema branching and act as a quorum sensing-like signal in the moss Physcomitrella patens. Development 138: 1531-1539.

Redecker D, Kodner R and Graham LE (2000). Glomalean fungi from the Ordovician. Science 289: 1920-1921.

Rensing SA, Ick J, Fawcett JA, Lang D, et al. (2007). An ancient genome duplication contributed to the abundance of metabolic genes in the moss Physcomitrella patens. BMC Evol. Biol. 7: 130.

Sanderson MJ and Doyle JA (2001). Sources of error and confidence intervals in estimating the age of angiosperms from rbcL and 18S rDNA data. Am. J. Bot. 88: 1499-1516.

Tamura K, Dudley J, Nei M and Kumar S (2007). MEGA4: Molecular Evolutionary Genetics Analysis (MEGA) software version 4.0. Mol. Biol. Evol. 24: 1596-1599.

Umehara M, Hanada A, Yoshida S, Akiyama K, et al. (2008). Inhibition of shoot branching by new terpenoid plant hormones. Nature 455: 195-200.

Vogel JT, Walter MH, Giavalisco P, Lytovchenko A, et al. (2010). S1CCD7 controls strigolactone biosynthesis, shoot branching and mycorrhiza-induced apocarotenoid formation in tomato. Plant J. 61: 300-311.

Wolfe KH, Gouy M, Yang YW, Sharp PM, et al. (1989a). Date of the monocot-dicot divergence estimated from chloroplast DNA sequence data. Proc. Natl. Acad. Sci. U. S. A. 86: 6201-6205.

Wolfe KH, Sharp PM and Li WH (1989b). Rates of synonymous substitution in plant nuclear genes. J. Mol. Evol. 29: 208-211. 
Yang Z (1998). Likelihood ratio tests for detecting positive selection and application to primate lysozyme evolution. Mol. Biol. Evol. 15: 568-573.

Yang Z (2007). PAML 4: phylogenetic analysis by maximum likelihood. Mol. Biol. Evol. 24: 1586-1591.

Yang Z and Nielsen R (2002). Codon-substitution models for detecting molecular adaptation at individual sites along specific lineages. Mol. Biol. Evol. 19: 908-917.

Yokota T, Sakaic H, Okunod K, Yoneyamad K, et al. (1998). Alectrol and orobanchol, germination stimulants for Orobanche minor, from its host red clover. Phytochemistry 49: 1967-1973.

Zhang J, Nielsen R and Yang Z (2005). Evaluation of an improved branch-site likelihood method for detecting positive selection at the molecular level. Mol. Biol. Evol. 22: 2472-2479. 